\title{
Experiência de cárie e fatores associados entre escolares das zonas urbana e rural: um estudo piloto
}

\author{
Caries experience and associated factors among urban and rural schoolchildren: a pilot study \\ Experiencia de caries y factores asociados entre escolares de las zonas urbanas y rurales: \\ un estudio piloto \\ Diego Romário SILVA \\ Sabrina Avelar de Macêdo FERREIRA ${ }^{2}$ \\ Pierre Andrade Pereira OLIVEIRA ${ }^{3}$ \\ Andréa Cristina Barbosa SILVA ${ }^{3}$ \\ ${ }^{I}$ Doutorando, Faculdade de Odontologia de Piracicaba - FOP/UNICAMP, Área de Farmacologia, Anestesiologia e Terapêutica \\ ${ }^{2}$ Cirurgiã-Dentista pela Universidade Estadual da Paraíba, UEPB \\ ${ }^{3}$ Professor(a) Doutor(a), Universidade Estatual da Paraíba - UEPB Campus VIII, Área de Saúde Coletiva e Odontologia Preventiva
}

\begin{abstract}
Resumo
Introdução: A cárie é uma doença infecciosa, de caráter multifatorial, dependente da dieta, microbiota bucal e resposta do hospedeiro, resultando na desmineralização localizada dos tecidos dentários duros. Objetivo: Avaliar a experiência de cárie e fatores associados em escolares residentes em zonas rurais e urbanas de Araruna, PB. Material e método: Um levantamento epidemiológico de prevalência de cárie dentária e alterações gengivais foi realizado. Os dados foram anotados em fichas odontológicas apropriadas, visando identificar diferenciais entre estes indicadores das duas áreas estudadas. Além disto, o fluxo salivar dos participantes foi determinado. Resultado: Os resultados indicaram diferenças brandas entre os indicadores de saúde bucal nas duas zonas. Apesar da tendência destes indicadores, como o CPOD, ISG e IHOS terem sido mais baixos na zona rural, há alguns anos atrás, do que os observados na zona urbana, no presente trabalho não foram identificadas diferenças significativas entre estes índices. Conclusão: A população rural vem obtendo acesso aos serviços encontrados na cidade, facilitando a inclusão na dieta de alimentos mais cariogênicos e, consequentemente, igualando os índices de saúde bucal aos encontrados na população urbana. Desta forma, o risco de cárie nestas populações, atualmente, é similar.
\end{abstract}

Descritores: Cárie Dentária; Epidemiologia; Zona Rural; Área Urbana.

\section{Abstract}

Introduction: The caries is an infectious, multifactorial disease, dependent on diet, oral microbiota and host response, resulting in localized demineralization of hard dental tissues. Objective: To evaluate the experience of caries and associated factors in schoolchildren residing in rural and urban areas of Araruna, PB. Material and method: An epidemiological survey of the prevalence of dental caries and gingival alterations was performed. The data were annotated in appropriate dental records, in order to identify differences between these indicators of the two areas studied. In addition, the participants' salivary flow was determined. Result: The results indicated mild differences between the oral health indicators in the two areas. Despite the tendency of these indicators, such as CPOD, ISG and IHOS to be lower in rural areas, a few years ago, in the present study, no significant differences were found between these indices. Conclusion: The rural population has been gaining access to the services found in the city, facilitating the inclusion of more cariogenic foods in the diet and, consequently, matching the oral health indexes to those found in the urban population. Thus, the risk of caries in these populations is currently similar.

Descriptors: Dental Caries; Epidemiology; Rural Áreas; Urban Área.

\section{Resumen}

Introducción: La caries es una enfermedad infecciosa, de carácter multifactorial, dependiente de la dieta, microbiota bucal y respuesta del huésped, resultando en la desmineralización localizada de los tejidos dorsales duros. Objetivo: Evaluar la experiencia de caries y factores asociados en escolares residentes en zonas rurales y urbanas de Araruna, PB. Material y método: Se realizó un levantamiento epidemiológico de prevalencia de caries y cambios en las encías. Los datos fueron anotados en fichas odontológicas apropiadas, buscando identificar diferenciales entre estos indicadores de las dos áreas estudiadas. Además, el flujo salivar de los participantes fue determinado. Resultado: Los resultados indicaron diferencias blandas entre los indicadores de salud bucal en las dos zonas. A pesar de la tendencia de estos indicadores, como el CPOD, ISG e IHOS han sido más bajos en la zona rural, hace algunos años, que los observados en la zona urbana, en el presente trabajo no se identificaron diferencias significativas entre estos índices. Conclusión: La población rural viene obteniendo acceso a los servicios encontrados en la ciudad, facilitando la inclusión en la dieta de alimentos más cariogénicos y, consecuentemente, igualando los índices de salud bucal a los encontrados en la población urbana. De esta forma, el riesgo de caries en estas poblaciones, actualmente, es similar.

Descriptores: Caries Dental; Epidemiología; Médio Rural; Área Urbana.

\section{INTRODUÇÃO}

A cárie dentária é uma doença complexa causada por uma desarmonia no equilíbrio fisiológico entre os minerais do dente e o fluido do biofilme ${ }^{1}$. Trata-se de uma doença infecciosa, localizada, de caráter multifatorial, dependente da dieta, microbiota bucal e resposta do hospedeiro, resultando na desmineralização dos tecidos dentários duros ${ }^{2}$.

Risco significa a probabilidade futura de que algo aconteça. Risco real de cárie é quando uma pessoa corre o risco de desenvolver lesões cariosas em determinada época ${ }^{3}$. Para saber se determinada doença possui um risco maior ou menor de ocorrer, é necessária a avaliação de fatores e indicadores de risco.
Os fatores de risco são aqueles diretamente envolvidos nos eventos bioquímicos, como uma grande quantidade de biofilme, dieta rica em carboidratos, baixa capacidade de tamponamento, redução do fluxo salivar e a presença de fluoretos, auxiliando na remineralização. Os indicadores de risco são os que agem de forma indireta, como fatores socioeconômicos, relacionados à saúde geral, epidemiológicos e clínicos ${ }^{4}$.

O Streptococcus mutans, uma bactéria Grampositiva, acidúrica e acidogênica, e capaz de se organizar em biofilme, é considerado o microrganismo mais associado à cárie ${ }^{5}$. A saliva pode influenciar o risco à cárie dentária por meio da 
remoção de microorganismos cariogênicos da boca, pelo fluxo ou por sua capacidade de aglutinar bactérias $^{6}$. Dessa forma, o fluxo salivar e a capacidade tampão são itens de extrema importância na prevenção e combate ao desafio cariogênico.

Considerando a escassez de informações e as limitações metodológicas dos estudos sobre este tema, faz-se necessário o desenvolvimento de pesquisas sobre cárie dentária nestas populações, com a finalidade de implementação, em curto e longo prazo, de políticas de saúde pública.

\section{MATERIAL E MÉTODO}

\section{Seleção dos participantes da pesquisa}

A seleção dos participantes da pesquisa, o preenchimento das fichas, os exames clínicos e a coleta das amostras foram realizados em escolas das zonas rurais e urbanas de Araruna, que foram selecionadas aleatoriamente para a pesquisa. Devido a impossibilidade de contato com alguns responsáveis pelas crianças nas escolas, alguns participantes das pesquisa foram avaliados em casa, na presença de seus responsáveis. Os testes salivares foram realizados no Laboratório de Microbiologia (Campus VIII, UEPB).

\section{Definição dos participantes da pesquisa}

O grupo de estudo consistiu de 20 crianças selecionadas aleatoriamente nas escolas. As informações coletadas e exames realizados foram anotados em fichas odontológicas contendo dados de identificação, CPO-D (dentes cariados perdidos e obturados) e ceo-d (dentes decíduos cariados, com extração indicada e obturados), de acordo com os seguintes critérios de elegibilidade de inclusão: 1) crianças de 4 - 10 anos (por possuírem uma microbiota mais diversa que a de adultos, e por apresentarem um comportamento mais favorável para a realização dos diversos exames que as de idade inferior); 2) que estudem nas escolas selecionadas (por possuírem uma dieta similar e padrão similar de escovação dentária); 3) que exibam padrão de alta atividade de cárie dentária (AAC), ou seja, que apresentem alto CPO-D e/ou cáries ativas) ou de baixa atividade de cárie dentária (BAC) com CPO-D $=0$ (cárie zero). Algumas crianças foram examinadas em suas residências, devido à necessidade de presença dos pais para a coleta dos dados sócioeconômicos e assinatura dos termos de consentimento livre e esclarecido. Os critérios de exclusão foram crianças que estivessem fazendo uso de qualquer tipo de administração medicamentosa, tópica, oral ou parenteral; e aquelas cujos responsáveis não autorizaram a participação na pesquisa.

\section{Preenchimento das fichas e exames clínicos}

Antes da coleta das amostras, os responsáveis pelas crianças foram entrevistados e os dados anotados em fichas contendo identificação do paciente, histórico odontológico, hábitos de higiene, hábitos alimentares, hábitos nocivos e exame clínico, com a obtenção dos Índices de Higiene Oral Simplificado (IHO-S), de Sangramento Gengival (ISG), CPO-D e ceo-d.

\section{Coleta das amostras salivares}

Para a determinação do fluxo salivar, a criança foi instruída a mascar um hiperbolóide por alguns segundos. Essa saliva foi deglutida por conter resíduos de alimentos. Depois a criança mascou o hiperbolóide durante 3 minutos, utilizando ambos os lados da boca. A saliva produzida neste período foi coletada em um tubo tipo Falcon ${ }^{\circledR}$ graduado acoplado a um funil de vidro, ambos estéreis. Em seguida, $1 \mathrm{~mL}$ desta saliva foi retirada, com o auxílio de uma pipeta de vidro estéril. A velocidade de fluxo salivar foi obtida dividindo-se a quantidade de saliva coletada pelo tempo. Os valores padrão segundo Groisman e Medeiros ${ }^{4}$ são: Normal: 1 a $2 \mathrm{ml} / \mathrm{min}$; Baixo: $<1 \mathrm{ml} / \mathrm{min}$; Xerostomia: $<0,2 \mathrm{ml} / \mathrm{mim}$.

\section{Análise estatística}

Os dados obtidos nesta pesquisa foram tabulados em programa SPSS v. 18.0. A análise estatística foi do tipo descritiva com apresentação de dados percentuais de frequência e média. Para a análise bi-variada foi utilizado o teste exato de Fisher para as variáveis qualitativas e o teste ANOVA para as variáveis quantitativas, com o nível de significância de 5\%.

\section{Aspectos éticos}

O projeto foi submetido ao Comitê de Ética em Pesquisa da UEPB para análise, sendo aprovado (número do parecer: 45063215.0.0000.5187). Termos de Consentimento Livre e Esclarecido e de Assentimento foram outorgados pelos responsáveis e escolares, respectivamente.

\section{RESULTADOS E DISCUSSÃO}

Neste estudo foi analisado um total de 20 crianças em idade escolar, das zonas urbana (10) e rural (10) da cidade de Araruna, PB. Foram avaliadas as seguintes variáveis: sexo, idade, zona, visita ao dentista, frequência de escovação, quem escova, uso de fio dental, hábitos deletérios, ISG, IHOS, CPO-D e fluxo salivar, para verificar a possibilidade de haver diferença na experiência e risco de cárie de um mesmo município, mas em áreas diferentes.

A idade das crianças examinadas variou de 5 a 10 anos, sendo 11 (45\%) do gênero feminino e 9 $(55 \%)$ do gênero masculino, como mostrado na Tabela 1. Na área urbana foram avaliadas $40 \%$ do sexo masculino e $60 \%$ do sexo feminino e na área rural $70 \%$ masculino e $30 \%$ feminino, como descrito na Tabela 2.

Com relação ao índice de hábitos deletérios, foi observado que $60 \%$ das crianças examinadas não apresentavam nenhum tipo de hábito, segundo exame 
clínico realizado e relato de seus responsáveis. Foi observado bruxismo em 5\%, mordedura de objetos em $10 \%$, respiração bucal em $10 \%$, onicofagia em $5 \%$, sucção digital em $5 \%$ e uso de chupeta também em 5\%, como mostra a Tabela 3.

Tabela 1. Descritivo da amostra de escolares das Zonas Rural e Urbana de Araruna, PB, 2014

\begin{tabular}{lcc}
\hline Variáveis & N & \% \\
\hline Sexo & & \\
Masculino & 11 & 55 \\
Feminino & 9 & 45 \\
Idade & & \\
5 a 8 anos & 12 & 60 \\
9 e 10 anos & 8 & 40 \\
Zona & & \\
Urbana & 10 & 50 \\
$\quad$ Rural & 10 & 50 \\
Dentista & & \\
Não & 2 & 10 \\
Sim & 18 & 90 \\
Escova & & \\
Sim & 20 & 100 \\
Quem & & \\
Pessoa & 11 & 55 \\
Mãe & 5 & 25 \\
Ambos & 4 & 20 \\
Vezes & & \\
1 & 7 & 35 \\
2 & 5 & 25 \\
3 & 8 & 40 \\
Fio & & \\
Não & 20 & 100 \\
\hline Total & $\mathbf{2 0}$ & $\mathbf{1 0 0}$ \\
\hline Fonte: Pesquisa direta, 2014 & & \\
\hline
\end{tabular}

Tabela 2. Distribuição do sexo da amostra por zona urbana e rural nos escolares de Araruna, PB, 2014

\begin{tabular}{lcccc}
\hline Variáveis & \multicolumn{4}{c}{ Sexo } \\
& N & Masculino & Feminino & Valor de p \\
\hline Zona & & & & \\
Urbana & 10 & $40 \%$ & $60 \%$ & $\mathbf{0 , 1 8 5 *}$ \\
Rural & 10 & $70 \%$ & $30 \%$ & \\
*Teste exato de Fisher \\
Fonte: Pesquisa direta, 2014
\end{tabular}

Tabela 3. Descritivo dos Hábitos Deletérios nos escolares das Zonas Rural e Urbana de Araruna, PB, 2014

\begin{tabular}{lcc}
\hline Variáveis & $\mathrm{N}$ & $\%$ \\
\hline Hábitos & 12 & 60 \\
0 & 1 & 5 \\
1 & 2 & 10 \\
2 & 2 & 10 \\
3 & 1 & 5 \\
4 & 1 & 5 \\
5 & 1 & 5 \\
6 & $\mathbf{2 0}$ & $\mathbf{1 0 0}$ \\
\hline Total &
\end{tabular}

O valor médio dos índices ISG, IOHS e CPO-D foram 3,28, 1,79 e 5,29, respectivamente, e estão representados na Tabela 4. De acordo com a zona analisada, os valores são mostrados na Tabela 5, sendo o ISG, IHOS, CPO-D e fluxo salivar 4,26,
$1,60,5,50,0,31$, respectivamente. Já na zona rural, os valores médios foram para ISG, IHOS, CPO-D e fluxo salivar, respectivamente, $2,30,1,82,6,40 \mathrm{e}$ 0,33 .

Tabela 4. Médias da amostra do Índice de Sangramento Gengival (ISG), Índice de Higiene Oral Simplificado (IHOS) e Dentes Cariados, Perdidos ou Obturados (CPO-D) nos escolares das Zonas Rural e Urbana de Araruna, PB, 2014

\begin{tabular}{lccc}
\hline Variáveis & N & Média & Desvio Padrão \\
\hline ISG & 20 & 3,28 & $\pm 3,06$ \\
IHOS & 19 & 1,70 & $\pm 0,61$ \\
CPO-D & 20 & 5,95 & $\pm 3,36$ \\
*Teste Estatístico ANOVA & \\
Fonte: Pesquisa direta, 2014
\end{tabular}

Tabela 5. Médias do Índice de Sangramento Gengival (ISG), Índice de Higiene Oral Simplificado (IHOS), Dentes Cariados, Perdidos ou Obturados (CPO-D) e Fluxo Salivar das Zonas Rural e Urbana de Araruna, PB, 2014

\begin{tabular}{|c|c|c|c|c|}
\hline Variável & ISG & IHOS & CPO-D & Fluxo \\
\hline \multicolumn{5}{|l|}{ Zona } \\
\hline Urbana & $\begin{array}{r}4,26 \\
( \pm 3,84)\end{array}$ & $\begin{array}{c}1,60 \\
( \pm 0,76)\end{array}$ & $\begin{array}{c}5,50 \\
( \pm 3,59)\end{array}$ & $\begin{array}{c}0,31 \\
( \pm 0,12)\end{array}$ \\
\hline Rural & $\begin{array}{r}2,30 \\
( \pm 1,69) \\
\end{array}$ & $\begin{array}{c}1,82 \\
( \pm 0,41) \\
\end{array}$ & $\begin{array}{c}6,40 \\
( \pm 3,23) \\
\end{array}$ & $\begin{array}{c}0,33 \\
( \pm 0,19) \\
\end{array}$ \\
\hline $\begin{array}{l}\text { Valor } \\
\text { de p }\end{array}$ & $p=0,158$ & $p=0,449$ & $p=0,564$ & $p=0,768$ \\
\hline
\end{tabular}

*Teste Estatístico ANOVA

Fonte: Pesquisa direta, 2014

Com relação à visita ao dentista (Tabela 6) e aos hábitos de higiene oral (Tabela 7), foi verificado que $80 \%$ das crianças da zona urbana e $100 \%$ das crianças da zona rural foram ao dentista e que apenas $40 \%$ de cada zona escovam os dentes 3 vezes ao dia, que é o recomendado.

Tabela 6. Distribuição da visita ao dentista por zona urbana e rural nos escolares de Araruna, PB, 2014

\begin{tabular}{lcccc}
\hline \multirow{2}{*}{ Variáveis } & \multicolumn{4}{c}{ Visita ao Dentista } \\
& N & Não & Sim & Valor de p \\
\hline Zona & & & & \\
Urbana & 10 & $20 \%$ & $80 \%$ & $\mathbf{0 , 2 3 7 *}$ \\
Rural & 10 & 0 & $100 \%$ & \\
*Teste Exato de Fisher & & & \\
Fonte: Pesquisa direta, 2014 & &
\end{tabular}

Tabela 7. Distribuição da quantidade de vezes que as crianças escovam os dentes por zona urbana e rural nos escolares de Araruna, PB, 2014

\begin{tabular}{lccccc}
\hline \multicolumn{5}{c}{ Quantas Vezes } & \multirow{2}{*}{ Valor de p } \\
& N & Uma & Duas & Três & \\
\hline Zona & & & & & \\
Urbana & 10 & $30 \%$ & $30 \%$ & $40 \%$ & $\mathbf{1 , 0 0 *}$ \\
Rural & 10 & $40 \%$ & $20 \%$ & $40 \%$ & \\
*Teste Exato de Fisher \\
Fonte: Pesquisa direta, 2014
\end{tabular}

Além disso, foi visto que na zona urbana $50 \%$ das crianças realizam a própria escovação, $30 \%$ quem realiza é o responsável e $20 \%$ ambos. Na zona urbana $60 \%$ das crianças escovam sozinhas, $20 \%$ 
quem escova é o responsável e os outros $20 \%$ ambos escovam, como mostra a Tabela 8.

Tabela 8. Distribuição de quem escova os dentes da criança por zona urbana e rural nos escolares de Araruna, PB, 2014.

\begin{tabular}{|c|c|c|c|c|c|}
\hline \multirow{2}{*}{ Variáveis } & \multicolumn{4}{|c|}{ Quem Escova } & \multirow{2}{*}{ Valor de I } \\
\hline & $\mathbf{N}$ & Pessoa & Mãe & Ambos & \\
\hline \multicolumn{6}{|l|}{ Zona } \\
\hline Urbana & 10 & $50 \%$ & $30 \%$ & $20 \%$ & $1,00 *$ \\
\hline Rural & 10 & $60 \%$ & $20 \%$ & $20 \%$ & \\
\hline
\end{tabular}

*Teste Exato de Fisher

Fonte: Pesquisa direta, 2014

Muitos autores têm pesquisado, por meio de variáveis clínicas e/ou microbiológicas, índices que mostrem risco e prevalência de cárie em escolares de áreas urbanas e rurais dos municípios, a fim de analisar se há diferenças significativas na experiência de cárie nas respectivas áreas.

Geus et al. $^{7}$ realizaram um delineamento transversal com 293 crianças da zona rural e 412 da zona urbana, no município de Ponta Grossa (PR). Os resultados mostraram que houve maior prevalência de cárie nas crianças das escolas rurais $(86,7 \%)$ do que das urbanas $(63,1 \%), \mathrm{p}<0,001$. Também houve maior proporção de relato de dor de dente na zona rural $(68,9 \%$ contra $59,9 \%, \mathrm{p}=0,014)$. O CPO-D médio desta região foi de 1,0 e o ceo-d foi de 3,87 . $\mathrm{Na}$ zona urbana, os índices CPO-D e ceo-d foram de 0,39 e 2,19 , respectivamente $(\mathrm{p}<0,001)$. Os índices ceo-d e CPO-D, em escolares residentes no meio urbano e rural de Ponta Grossa, foram diferentes, sendo significativamente maiores para a zona rural. Além disso, prevalência de cárie severa também foi maior na zona rural.

No presente estudo, o índice de CPO-D foi maior na zona rural $(6,40)$ do que na urbana $(5,50)$, mostrando uma pequena diferença na experiência de cárie entre as duas áreas, sendo a zona rural com o índice um pouco maior. Entretanto, em se tratando do índice ISG e IOHS a situação se inverte, sendo o ISG da zona urbana 4,26 e da rural 3,30, e o IHOS 1,60 e 1,82 , respectivamente.

Silva et al. $^{8}$ estudaram sete escolas localizadas na área urbana e seis na área rural de Vassouras (RJ). Os resultados mostraram que, aos 12 anos, a prevalência de cárie medida pelo índice CPO$\mathrm{D}$ encontrava-se menor nas áreas urbanas $(1,54)$ que nas rurais $(3,18)$ destacando a correlação existente entre a distância dos centros de atendimento e a alta prevalência da doença. Todavia, apesar da justificativa do estudo, para a diferença no índice CPO-D, ser a distância da população rural dos centros de atendimento, no presente estudo verificouse que o número de crianças da zona rural que frequentaram o dentista é superior ao da zona urbana. A explicação para essa discrepância pode estar no modo de vida da população e de administração da saúde pública nos diferentes estados da pesquisa.
Em 2009, Silva et al. ${ }^{9}$ observaram que em Igaratinga (MG) o número de dentes restaurados tanto na dentição decídua quanto na permanente foi maior na zona urbana do que na rural. Entretanto, segundo os autores, não foi provado se estes resultados foram obtidos devido a alta experiência de cárie ou a facilidade de acesso ao tratamento, que os pacientes da zona urbana teriam em relação aos da zona rural.

\section{CONCLUSÃO}

Apesar da tendência dos indicadores de saúde bucal, como o CPOD, ISG e IHOS se mostrarem mais baixos na zona rural quando comparados à zona urbana, no presente trabalho não foram identificadas diferenças significativas entre estes índices. Estes resultados indicam que cada vez mais, a população rural vem obtendo acesso aos serviços encontrados na cidade, facilitando a inclusão na dieta de alimentos mais cariogênicos e, consequentemente, igualando seus índices de saúde bucal aos encontrados na população urbana. Assim, o risco de cárie se mostra similar nestas populações.

\section{REFERÊNCIAS}

1. Fejerskov O, Nyvad B. Is dental caries an infections disease? Diagnostic and treatment consequences for the practitioner; in Schou L (ed): Nordic Dentistry 2003 Yearbook. Copenhagen, Quintessence Publishing, p.141$151 ; 2003$.

2. $\mathrm{Xu} X, \mathrm{Z}$, hou XD, WU CD. The tea catechin epigallocatechin gallate suppresses cariogenic factors of streptococcus mutans. Antimicrob Agents Chemother. 2011; 55(3):1229-36.

3. Krasse B. Risco de cárie: um guia prático para avaliação e controle. Tradução; José Luiz Freire de Andrade. São Paulo: Quintessence; 1988.

4. Groisman S, Medeiros U. Cariologia e a Clínica. In: KRIGER L. Promoção de Saúde Bucal. 3.ed. São Paulo: Artes Médicas, p.69-87; 2003.

5. Loesche WJ. Role of Streptococcus mutans in human dental decay. Microbiol Rev. 1986; 50(4):353-80.

6. Herrera JL, Lyons MF 2nd, Johnson LF. Saliva: its role in health and disease. $\mathrm{J}$ Clin Gastroenterol. 1988; 10(5):569-78.

7. Geus JL, Luca CMB, Baldani MH, Czlusniak GD. Prevalência de cárie e autopercepção da condição de saúde bucal entre crianças de escolas urbanas e rurais de Ponta Grossa - PR. Pesq Bras Odontoped Clin Integr. 2013; 13(1):111-17.

8. Silva MAM, Souza MCA, Rodrigues CRT, Bello RF. Condições de saúde bucal em escolares de Vassouras/RJ: uma pesquisa epidemiológica. Rev bras pesqui saúde. 2010; 12(15):52-6.

9. Silva AM, Vargas AMD, Ferreira EF. Diferenças na experiência de cárie dental em crianças residentes em áreas urbanas e rurais. Arq Odont. 2009; 45(3):122-28. 


\section{CONFLITO DE INTERESSES}

Os autores declaram não haver conflitos de interesse.

\section{AUTOR PARA CORRESPONDENCIA}

\section{Diego Romário Silva}

diegoromarioo@gmail.com

Submetido em 12/06/2018

Aceito em 23/08/2018 